\title{
The efficacy of inhaled nanoparticle tacrolimus in preventing rejection in an orthotopic rat lung transplant model
}

\author{
Nitin Andrew Das, MD, ${ }^{\mathrm{a}}$ Jay I. Peters, MD, ${ }^{\mathrm{d}}$ Jeremy D. Simmons, MD, ${ }^{\mathrm{a}}$ Yibo Wang, PhD, \\ Kevin P. O'Donnell, PhD, ${ }^{\mathrm{e}}$ Daniel T. DeArmond, MD, ${ }^{\mathrm{a}}$ Jacqueline J. Coalson, MD, \\ Edward G. Brooks, MD, ${ }^{\mathrm{c}}$ and Scott B. Johnson, $\mathrm{MD}^{\mathrm{a}}$
}

\begin{abstract}
Objective: The immunosuppressive efficacy of inhaled nanoparticle tacrolimus was compared with systemic tacrolimus in a rodent allogeneic lung transplant model.

Methods: Sixteen rats underwent allogeneic left orthotopic lung transplantation and were divided into 3 treatment groups: (1) inhaled nanoparticle tacrolimus: $6.4 \mathrm{mg}$ tacrolimus $/ 6.4 \mathrm{mg}$ lactose twice per day; (2) intramuscular tacrolimus: $1 \mathrm{mg} / \mathrm{kg}$ tacrolimus once per day; and (3) inhaled lactose: $6.4 \mathrm{mg}$ of lactose twice per day. Five days after transplant, the rats were necropsied and underwent histologic rejection grading and cytokine analysis. Trough levels of tacrolimus were measured in allograft, blood, and kidney.
\end{abstract}

Results: Both intramuscular $(\mathrm{n}=6)$ and nanoparticle tacrolimus $(\mathrm{n}=6)$ rats displayed lower histologic grades of rejection (mean scores $3.4 \pm 0.6$ and $4.6 \pm 0.9$, respectively) when compared with lactose rats $(\mathrm{n}=4)$ (mean score $11.38 \pm 0.5$, $P=.07)$. Systemic tacrolimus trough levels (median) were lower in nanoparticle tacrolimus-treated rats versus intramuscular-treated rats $(29.2$ vs $118.6 \mathrm{ng} / \mathrm{g}$; $P<.001$ in kidney, and $1.5 \mathrm{vs} 4.8 \mathrm{ng} / \mathrm{mL} ; P=.01$ in blood).

Conclusions: Inhaled nanoparticle tacrolimus provided similar efficacy in preventing acute rejection when compared with systemic tacrolimus while maintaining lower systemic levels. (J Thorac Cardiovasc Surg 2017;154:2144-51)

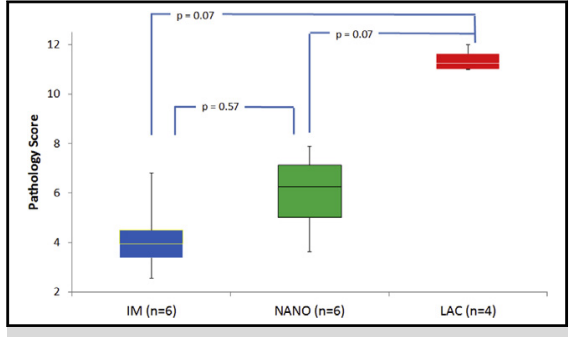

Box-whisker plot of pathology score in the treatment (IM and NANO) and control (LAC) groups.

\section{Central Message}

nTAC administered via inhalation shows similar attenuation of acute rejection in a rodent lung allograft as systemic therapy.

\section{Perspective}

Inhaled therapy of antirejection medication may provide adequate immunosuppression while maintaining low systemic levels and therefore less adverse toxic effects.

See Editorial Commentary page 2152.
According to the 2016 Registry of the International Society for Heart and Lung Transplantation, more than 3900 lung transplants were performed in 2014 and the overall median survival for lung transplant recipients is 5.8 years, with approximately $30 \%$ of patients experiencing an episode of acute rejection within the first year of transplant. ${ }^{1}$ These episodes of acute rejection are known risk factors for the

From the Departments of ${ }^{\mathrm{a} C}$ ardiothoracic Surgery, ${ }^{\mathrm{b}}$ Pathology, and ${ }^{\mathrm{c}}$ Pediatrics, and ${ }^{\mathrm{d}}$ Division of Pulmonology, Department of Medicine, University of Texas Health Science Center at San Antonio, San Antonio; and ${ }^{\mathrm{e} C o l l e g e ~ o f ~ P h a r m a c y, ~ U n i v e r s i t y ~}$ of Texas in Austin, Austin, Tex.

This study was funded in part by the Institute for Integration of Medicine and Science Clinical and Translational Science Award Grant UL1RR025767. The project described was supported by the National Institutes of Health's National Center for Research Resources and the National Center for Advancing Translational Sciences, through Grant 8UL1TR000149. The content is solely the responsibility of the authors and does not necessarily represent the official views of the National Institutes of Health.

Received for publication Feb 24, 2017; revisions received June 27, 2017; accepted for publication July 16, 2017; available ahead of print Sept 21, 2017.

Address for reprints: Nitin Andrew Das, MD, University of Texas Health Science Center at San Antonio, School of Medicine Building, 7703 Floyd Curl Dr, MC 7841 (Room 211 L), San Antonio, TX 78229 (E-mail: dasn@uthscsa.edu).

$0022-5223 / \$ 36.00$

Copyright (c) 2017 by The American Association for Thoracic Surgery

http://dx.doi.org/10.1016/j.jtcvs.2017.07.083 development of chronic rejection and bronchiolitis obliterans syndrome within a few years after lung transplantation. ${ }^{2,3}$ Although the strategies vary among transplant centers and continue to evolve, the mainstay for prevention of acute and chronic rejection remains systemic immunosuppression, with initial induction by a 3-drug regimen (calcineurin inhibitor or other interleukin [IL]-2 antagonists, in addition to corticosteroids and an antimetabolite), followed by maintenance therapy. ${ }^{4}$

Current therapy with tacrolimus (oral and intravenous) can cause systemic toxicity (especially renal) and increase the risk of opportunistic infections. Lungs offer a direct route for administering therapy by inhalation, which may circumvent some of the associated systemic toxicity and potentially achieve higher concentrations at the target site

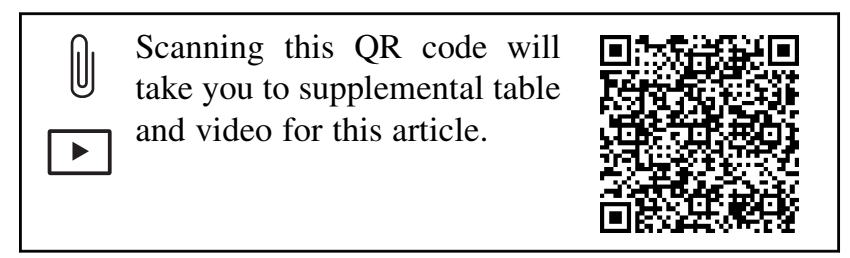




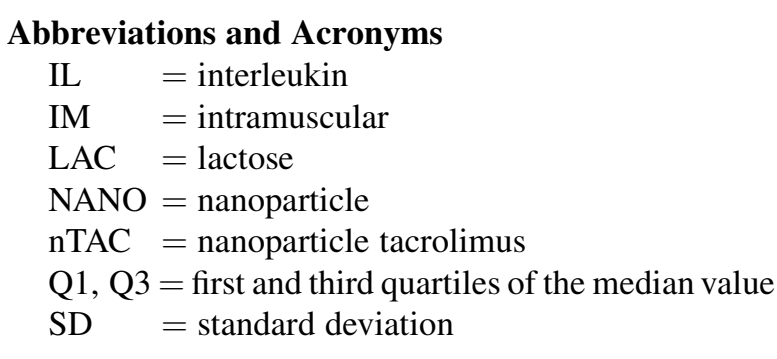

in lung transplant recipients. Prior attempts using aerosolized cyclosporine had limited success because of airway reactivity to the polyethylene glycol solvent in these formulations. ${ }^{5,6}$

A novel formulation of tacrolimus has been developed by the College of Pharmacy, University of Texas in Austin, prepared from anhydrous tacrolimus using a thin film freezing process and stabilized with lactose molecules. ${ }^{7}$ This brittle matrix formulation of nanoparticle tacrolimus (nTAC) provides greater aqueous solubility, a greater surface area relative to conventional tacrolimus, and a dispersion size less than $5 \mu \mathrm{m}$, theoretically enabling it to reach the lung's smallest and most distal airways and therefore behaving like a nanoparticle. ${ }^{7,8}$

Previous work using this nTAC in a single-dose nonrejection (syngeneic) orthotopic rat lung transplantation model confirmed satisfactory lung tissue levels with negligible systemic levels. $^{8}$

The objective of this study was to compare the antirejection efficacy of this novel inhaled nTAC with that of systemic (standard) tacrolimus in an allogeneic orthotopic rat lung transplantation model, to measure blood and tissue tacrolimus levels, and to perform cytokine analysis.

\section{MATERIALS AND METHODS}

All of the animals received humane treatment in accordance with the "Principles of Laboratory Animal Care" formulated by the National Society for Medical Research and the "Guide for the Care and Use of Laboratory Animals" prepared by the National Academy of Sciences and published by the National Institutes of Health, under a protocol approved by the Institutional Animal Care and Use Committee of The University of Texas Health Science Center at San Antonio (protocol number 10084x).

Tacrolimus monohydrate (Batch \#090703) was purchased from CQ International Co Inc (Cambridge, Mass). nTAC powder was produced as a 1:1 mixture with lactose by the College of Pharmacy, University of Texas at Austin, using a thin film freezing process described previously.

Allogeneic orthotopic left lung transplants were performed using inbred adult male Brown Norway rats as donors and inbred Lewis rats as recipients (Charles River, Wilmington, Mass) using the modified cuff technique previously described by Mizuta and associates.'

Donor rats were anesthetized using 3\% to 5\% inhaled isoflurane (Halocarbon, River Edge, $\mathrm{NJ}$ ) admixed with oxygen and were ventilated via tracheostomy using a Harvard Model 683 Small Animal Ventilator (Harvard Apparatus, Holliston, Mass) with a tidal volume of $10 \mathrm{~mL} / \mathrm{kg}$ at a rate of 60 to $80 \mathrm{breaths} / \mathrm{min}$ with positive end-expiratory pressure of $2 \mathrm{~cm}$ of water. Animals were dosed with heparin (APP Pharmaceuticals, Schaumburg,
Ill) 1000 units $/ \mathrm{kg}$ intravenously through the penile vein (alternatively into the hepatic bed) to achieve systemic heparinization over 5 minutes. The thoracic cavity was exposed via a generous laparotomy incision, and the lungs were flushed in anterograde fashion using cooled low potassium dextrose preservative solution, Perfadex (XVIVO, Goteborg, Sweden), to achieve uniform pallor of the lung graft. The entire heart-lung block was then excised, and the left hilum was dissected on a cold operating stage. The pulmonary vein, pulmonary artery, and main bronchus of the left lung were mounted with cuffs fashioned out of 14-gauge Angiocath (Becton Dickinson, Sandy, Utah). Lungs were stored at $4^{\circ} \mathrm{C}$ in a partially inflated state (end-tidal volume, $\sim 3-4 \mathrm{~cm}$ water pressure), immersed in the preservative solution.

After the explant, the recipient procedure was performed. Recipient rats were anesthetized, orally intubated using a $14 \mathrm{G}$ cannula, and ventilated with similar settings as the donor. Body temperature was maintained at approximately $36.5^{\circ} \mathrm{C}$ using a water-bath warmer. The left pulmonary hilum was exposed via a third intercostal thoracotomy, and the pulmonary artery, vein, and bronchus were individually clamped using microaneurysm clips (Yasargil; Aesculap, Center Valley, Pa). The donor pulmonary artery, vein, and bronchus were then aligned and telescoped into the respective structures of the recipient and anastomotic cuffs secured using 6-0 silk ties. After completion of the transplant, the recipient was allowed to recover from the effects of anesthesia in a warm chamber with $100 \%$ oxygen and given a single dose of $5 \mathrm{mg} / \mathrm{kg}$ enrofloxacin (Baytril; Bayer, Shawnee Mission, Kans) intramuscularly (IM) for infection prophylaxis.

\section{Grouping}

After transplantation, the recipients were divided into 1 of 3 groups, the lactose (LAC) group, IM group, and nanoparticle (NANO) group, as depicted in the flow diagram of experimental design (Figure 1).

The NANO group received nebulized nTAC administered every 12 hours by inhalation in a 4-port nose-only dosing chamber using the AeroNeb Pro nebulizer (Aerogen, Galway, Ireland). An amount of $12.8 \mathrm{mg}$ of nTAC powder $(6.4 \mathrm{mg}$ tacrolimus $+6.4 \mathrm{mg}$ lactose $)$ was dispersed in $6 \mathrm{~mL}$ of normal saline by sonication for 3 minutes for nebulization.

The LAC group was treated with nebulized lactose every 12 hours in a similar manner as nTAC in the NANO group. Lactose solution was prepared by mixing $6.4 \mathrm{mg}$ of lactose powder with $6 \mathrm{~mL}$ of normal saline by agitation for 3 minutes. The IM group received anhydrous tacrolimus suspended in normal saline and administered at a concentration of $1 \mathrm{mg} /$ $\mathrm{mL} / \mathrm{kg}$ of recipient body weight into the thigh once every 24 hours. ${ }^{10}$ The injected side was alternated each day. The first dose of the each medication was administered after the animals had recovered fully from anesthesia and breathing spontaneously.

\section{Experimental End Point}

The recipient animals were electively euthanized at postoperative day 5 by exsanguination through a cardiac puncture under deep anesthesia. The time of euthanasia was adjusted to coincide with the approximate time for their next treatment (the IM group was euthanized 24 hours after the final dose, whereas animals in the NANO and LAC groups were killed 12 hours after the final dose). Thus, the drug concentration levels in the blood and tissue would reflect trough levels of tacrolimus. At necropsy, allografts were examined for aeration and then divided into 3 coronal segments. The superior and middle segments of the allograft, and both kidneys from the recipient were snap-frozen in liquid nitrogen and stored at $-80^{\circ} \mathrm{C}$ for later analysis. The inferior segments were preserved in $10 \%$ buffered formalin for histologic examination. A portion of blood collected in ethylenediaminetetraacetic acid from cardiac puncture at the time of euthanasia was centrifuged, and the supernatant plasma was saved at $-80^{\circ} \mathrm{C}$ for tacrolimus level analysis. The remaining sample was saved as whole blood at $-80^{\circ} \mathrm{C}$ for later analysis. A brief video has been compiled to illustrate this methodology (Video 1). 


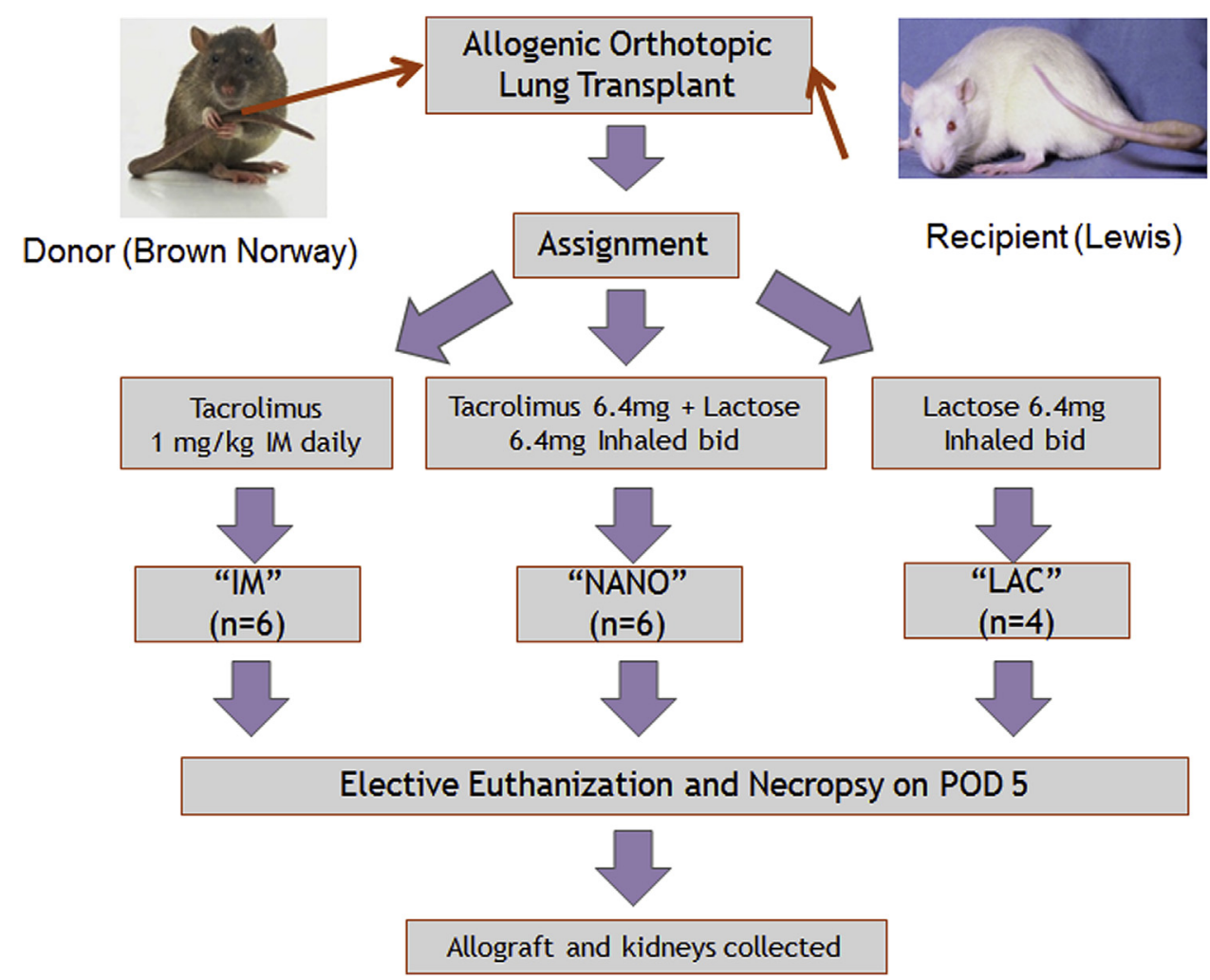

FIGURE 1. Flow diagram for experimental design depicting the group allocation and study time-points. IM, Intramuscular; NANO, nanoparticle; $L A C$, lactose; $P O D$, postoperative day.

\section{Histologic Rejection Grading}

The inferior segments of the allograft were embedded in paraffin, sectioned at $4 \mu \mathrm{m}$, and stained with hematoxylin-eosin. These samples were reviewed and graded by a pulmonary pathologist blinded to the experimental groups using 2 methods:

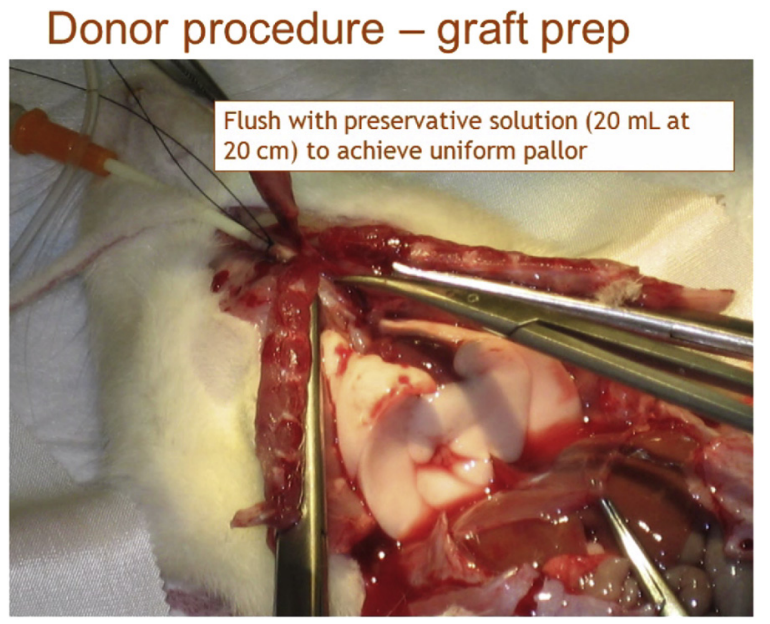

VIDEO 1. Short video (without audio) depicting the microsurgical procedure for left orthotopic lung transplant in rats and brief review of this study. Video available at: http://www.jtcvsonline.org/article/S00225223(17)31782-8/fulltext.
1. Revised 1996 Working Formulation for Standardization and Nomenclature in the Diagnosis of Lung Rejection by International Society for Heart and Lung Transplantation ${ }^{11}$ : Briefly, acute vascular rejection was graded as follows: A0-no lymphocytes around vessels, A1-small amount of lymphocytes around vessel, A2-vessel surrounded by lymphocytes, A3-lymphocytes extend into interstitial space, A4-lymphocytes overflow into surrounding alveoli. Acute airway rejection was graded as follows: B0R-no lymphocytes in airway, B1R-some lymphocytes in airway but within submucosa, B2R-lymphocytes extend beyond the basement membrane of the airway.

2. Modified Pathology Score: A second grading method was used to evaluate 4 anatomic sites-bronchial, bronchiolar, vascular, and alveolar space cellularity-separately. Scoring of each slide was done microscopically and graded using scores ranging from 0 to 3 to each region $(0=$ no inflammation, $1=$ mild inflammation, 2 = moderate inflammation, 3 = severe inflammation). The scores from these 4 regions were totaled to create an aggregate score ranging from 0 to 12 , with a score of zero implying no rejection and score of 12 reflecting fulminant graft rejection in all the regions examined. With the use of an Aperio image analysis system (Aperio, Inc, Sausalito, Calif), whole slide digital images of all study lungs were used to objectively document the extent and severity of pathologic findings.

\section{Tacrolimus Levels}

The levels of tacrolimus in the blood were measured using the PROTracTM II FK 506 enzyme-linked immunosorbent assay kit (Diasorin Inc, Stillwater, Minn) in batched assays. The levels of tacrolimus in the lungs and kidney were extracted by a 2 -step solvent extraction procedure 
using acetonitrile followed by ethyl acetate. The concentration of tacrolimus was quantified by liquid chromatography/mass spectrometry using Ascomycin as an internal standard. ${ }^{8}$

\section{Lung Allograft Cytokines}

Snap-frozen lung tissue (0.1-0.4 g) was thawed on ice and homogenized in $0.4 \mathrm{~mL}$ TPER (Pierce, Thermo Fisher Scientific Inc, Rockford, Ill) with 1 tablet/10 mL of Complete Mini Protease Inhibitor Cocktail (Roche Diagnostics, Mannheim, Germany) and supernatants collected after centrifugation. IL-1 $\alpha$, IL-1 $\beta$, IL-2, IL-4, IL-6, IL-10, IL-12 (p40/p70), granulocytemacrophage colony-stimulating factor, interferon- $\gamma$, and tumor necrosis factor- $\alpha$ were quantified by micro-bead array using a rat 10-plex panel (Invitrogen, Camarillo, Calif) analyzed on a Flexmap 3D (Luminex, Austin, Tex). Rat IL-17 and IL-18 were quantified using rat IL-17A (eBioscience, San Diego, Calif) and IL-18 (Invitrogen) enzyme-linked immunosorbent assays according to the manufacturers' instructions.

\section{Statistical Analysis}

Biomarker levels were log transformed in all analyses. Intergroup comparisons were accomplished using analysis of variance. $P$ values for pairwise comparisons were adjusted using the Kruskal-Wallis test with Hochberg correction. Pearson's product-moment correlation coefficient was calculated to determine the association between the logarithm of tacrolimus concentration and the lung pathology scores. All statistical analyses were performed using R 2.12.2 (R-Foundation).

\section{RESULTS}

All the animals that survived the transplant procedure were entered into the study and survived until day 5 when they were euthanized electively. No rats died during treatment, and a total of 16 rats were included in the study: $6 \mathrm{IM}, 6 \mathrm{NANO}$, and 4 LAC.

\section{Operative Characteristics}

The mean operative time for these animals $(\mathrm{n}=16)$ was $102.1 \pm 9$ minutes, and the mean ischemia time for the allograft was $82.2 \pm 10$ minutes (mean \pm standard deviation [SD]) with no significant differences between groups $(P=.29$ and $P=.65$, respectively). All recipients received their first dose of treatment after recovery from anesthesia within 100 minutes of reperfusion of the allograft.

Percent weight loss tended to be higher in the IM group compared with the NANO and LAC groups (mean \pm SD: $-10.7 \pm 0.75$ vs $-8.44 \pm 2.2$ and $-8.6 \pm 1.38 ; P=.06$ ), although this difference was not significant. No signs of respiratory distress were observed after animals received inhalation of tacrolimus.

\section{Histologic Findings}

With regard to allograft rejection, all LAC rats experienced severe AR4, B2R rejection, whereas all IM and NANO rats displayed AR2, B1R rejection on histopathologic evaluation. Mean aggregate modified path scores (mean \pm SD) for both IM $(3.4 \pm 0.6)$ and NANO $(4.6 \pm 0.9)$ were lower when compared with LAC $(11.38 \pm 0.5)$, approaching statistical significance

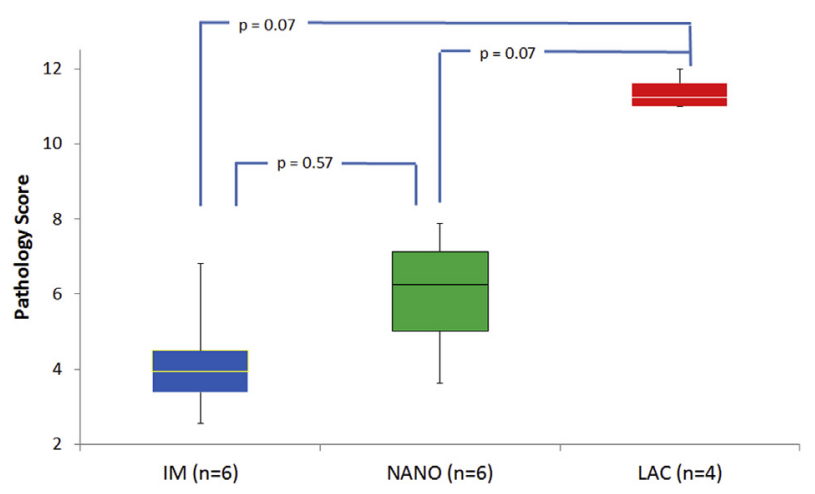

FIGURE 2. Box-whisker graph illustrating total pathology score in the treatment (IM and NANO) and control (LAC) groups. IM, Intramuscular; $N A N O$, nanoparticle; $L A C$, lactose.

$(P=.07)$. There was no difference when IM was compared with NANO $(P=.57)$, as shown in Figure 2. A detailed pathology scoring for each group is available for review as Table E1. On scanned digital images, the most striking difference was the loss of intra-alveolar inflammatory infiltrate in the lungs of the 2 treated (NANO and IM) groups when compared with the untreated (LAC) group (Figure 3, A$C)$. Microscopically, the LAC lungs were inflamed and had mixed cell populations of lymphoid (and other mononuclear cells), eosinophils, and neutrophils in airway and vascular compartments, with lymphoid cells being the dominant cell. Alveolar macrophages, monocytes, and neutrophils were the primary intra-alveolar cells. Peribronchial, peribronchiolar, and perivascular cellular infiltrates persisted in the treated IM and NANO groups on scanned digital images, but they histologically appeared less cellular and dense overall compared with the LAC lung (Figure 3, $D-F)$.

\section{Tacrolimus Levels}

The blood levels of tacrolimus in IM rats were significantly higher than in NANO rats (mean \pm SD: $5.4 \pm 3.4 \mathrm{ng} / \mathrm{mL}$ vs $1.4 \pm 0.3 \mathrm{ng} / \mathrm{mL}$, respectively; $P=.01)$. The mean tacrolimus levels in the kidneys were significantly higher in IM rats $(\mathrm{n}=6)$ compared with NANO rats $(\mathrm{n}=5)($ mean \pm SD: $121.8 \pm 57.7 \mathrm{ng} / \mathrm{g}$ vs $29.4 \pm 5 \mathrm{ng} / \mathrm{g} ; P<.001)$. Median allograft tissue tacrolimus levels were significantly higher in the systemically treated IM group $(\mathrm{n}=6)$ compared with the regionally treated NANO group $(\mathrm{n}=6)$ median $(\mathrm{Q} 1, \mathrm{Q} 3)$ : 764.9 (546.4, $1916.5) \mathrm{ng} / \mathrm{g}$ versus $154.4(153.5,160.6) \mathrm{ng} / \mathrm{g} ; P=.017)$.

\section{Cytokines}

The cytokine levels in the lung allograft were normalized by the weight of the tissue and expressed in nanograms/ gram. Of the analyzed cytokines, IL- $1 \alpha, \mathrm{IL}-1 \beta$, IL- 12 , IL17 , and IL-18 were quantifiable, and all others fell below assay limits of detection. With regard to IL- $1 \alpha$, IL- $1 \beta$, 


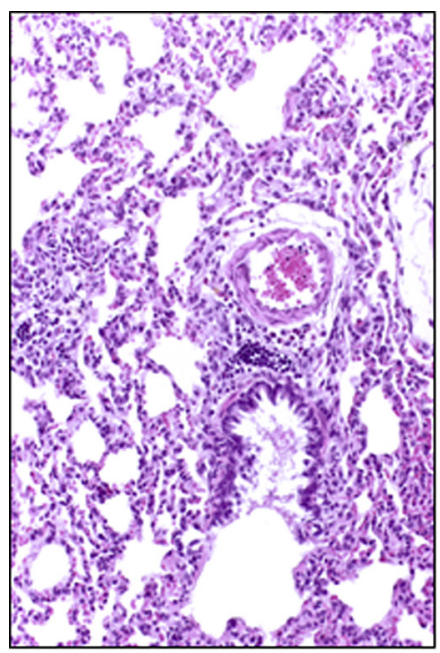

A $\operatorname{IM}(20 \mathrm{x})$

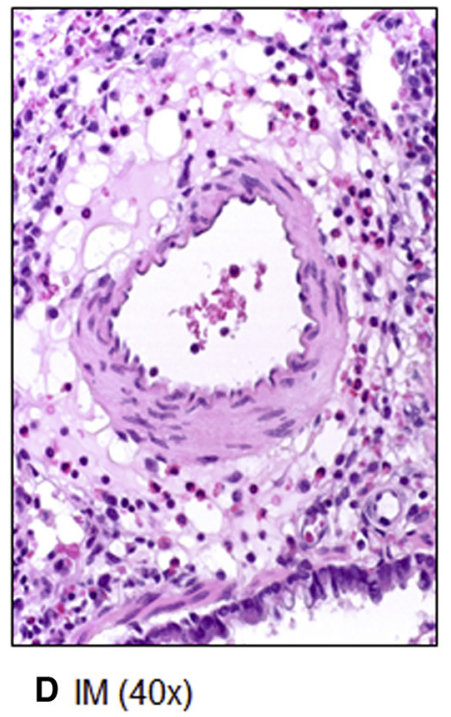

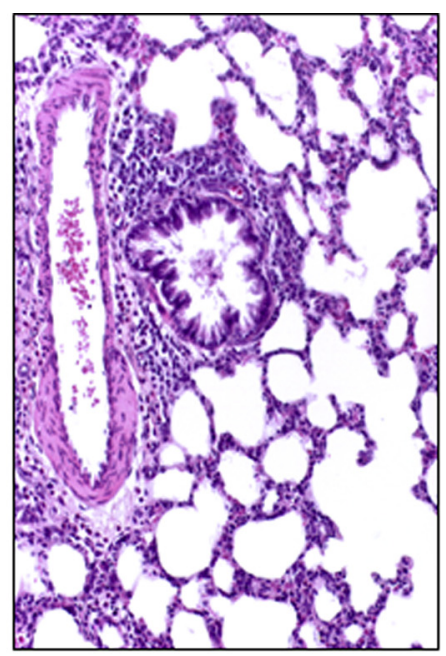

B NANO (20x)

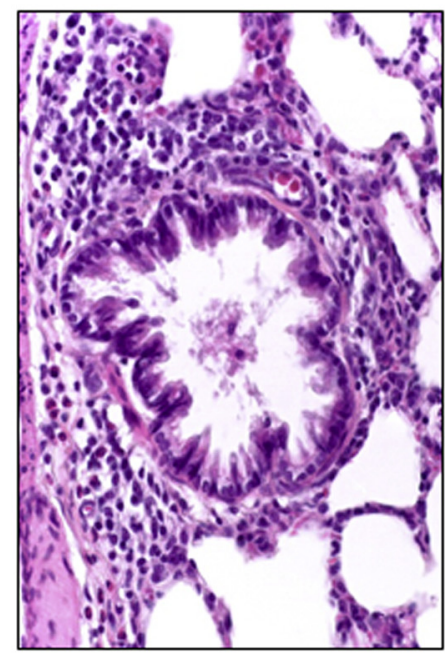

E $\operatorname{NANO}(40 \mathrm{x})$

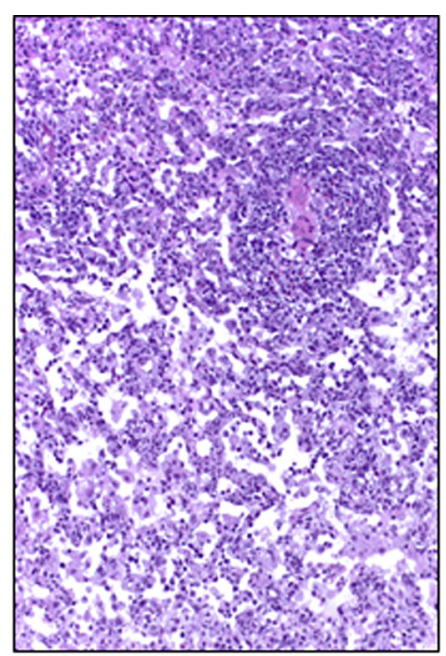

C $\operatorname{LAC}(20 \mathrm{x})$

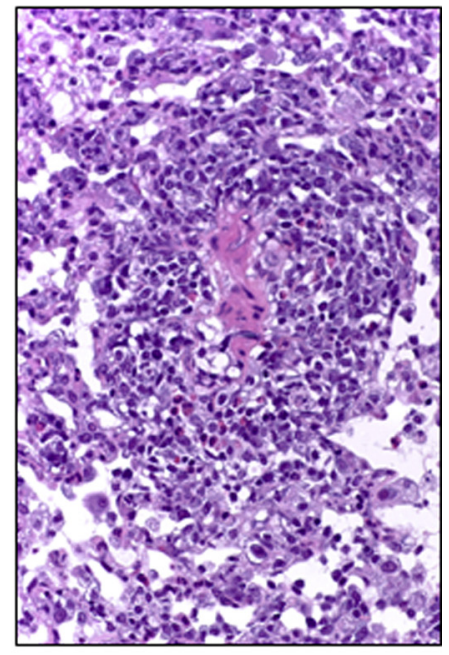

F $\operatorname{LAC}(40 \mathrm{x})$

FIGURE 3. Representative hematoxylin-eosin stained pictures at $20 \times(\mathrm{A}-\mathrm{C})$ and $40 \times(\mathrm{D}-\mathrm{F})$ magnification from lung allografts, 5 days after transplant showing mild to moderate rejection in the treatment groups (IM and NANO) and severe rejection in the control group (LAC). IM, Intramuscular; NANO, nanoparticle; $L A C$, lactose.

and IL-18, mean cytokine levels were significantly higher $(P \leq .001)$ among LAC rats (Figure 4$)$. There was no difference between IM and NANO. Mean IL-12 levels were significantly higher in NANO when compared with IM and LAC (Figure 5). There was no difference between IM and LAC. Mean IL-17 levels were significantly higher in IM when compared with NANO and LAC (Figure 6).

\section{DISCUSSION}

This study is unique in its evaluation of a novel formulation of tacrolimus that can be delivered by inhalation. This brittle matrix formulation made by thin-film freezing reduces the particle size of the drug to approximately $200 \mathrm{~nm}$, and this allows the drug to have pharmacologic properties of a nanoparticulate molecule. Theoretically, it has the potential for dispersion into more distal and smaller airways. In contrast, Ide and colleagues ${ }^{10}$ used the standard form of the drug in their study.

This inhalable formulation differs from other attempts at delivering nebulized immunosuppression directly to the lungs by use of lactose as a stabilizer and avoiding the use of noxious solvents such as polyethylene glycol, ${ }^{5}$ thus making this drug more tolerable and potentially improved treatment compliance in the lung transplant recipients. ${ }^{12}$

Bayer and colleagues ${ }^{13}$ also showed the beneficial effect of inhaled tacrolimus formulation in attenuating acute ischemia-reperfusion injury when administered to the donor lung before organ procurement. 

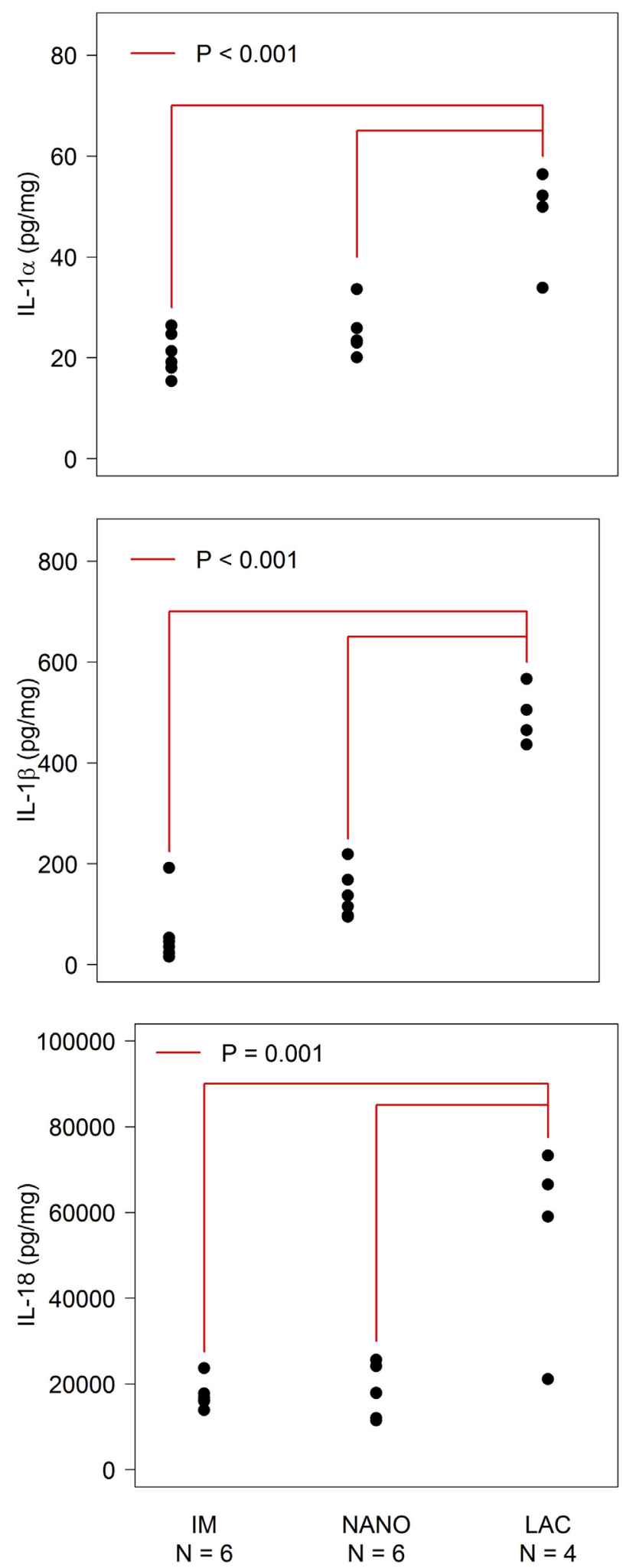

FIGURE 4. Scatter plot graphs depicting reduction of proinflammatory cytokines (IL-1 $\alpha, \mathrm{IL}-1 \beta$, and IL-18) in the treatment group (IM and NANO). $I L$, Interleukin; $I M$, intramuscular; $N A N O$, nanoparticle; $L A C$, lactose.

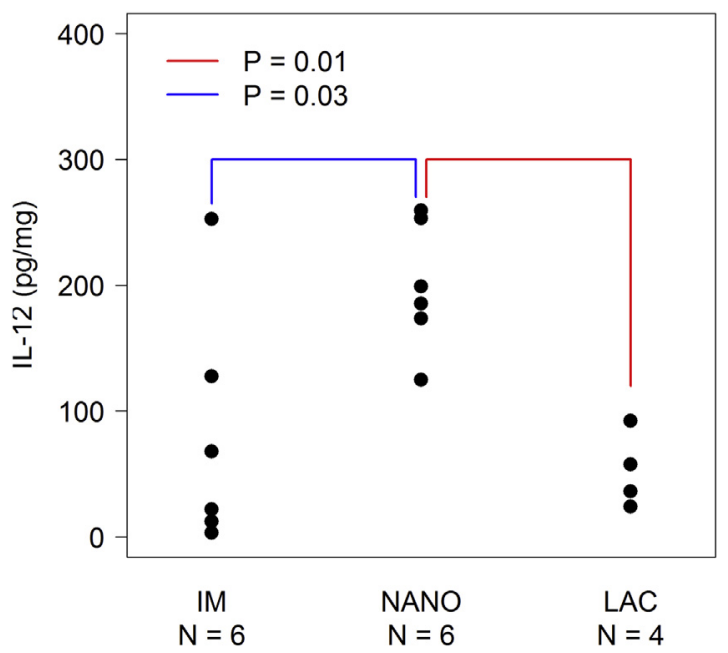

FIGURE 5. Scatter plot graph illustrating elevated IL-12 levels in the NANO group but lower levels in the IM and LAC groups. IL, Interleukin; $I M$, intramuscular; $N A N O$, nanoparticle; $L A C$, lactose.

In this study, we were able to achieve similar attenuation of rejection with inhalable tacrolimus (NANO) as the systemic IM treatment (Figure 2). An evaluation of tissue drug concentration shows significantly lower levels of tacrolimus in the kidneys of the inhaled tacrolimus group (Figure 7, left) and thereby reduced potential for occurrence of adverse side effect, such as nephrotoxicity or other systemic toxicity. Our study supports the finding by Watts and colleagues ${ }^{8}$ that minimal amounts of drug are absorbed in the systemic circulation after inhalation with this formulation of tacrolimus. For this study, we sought a peak lung concentration level of at least $270.4 \mathrm{ng} / \mathrm{g}$ in the allograft

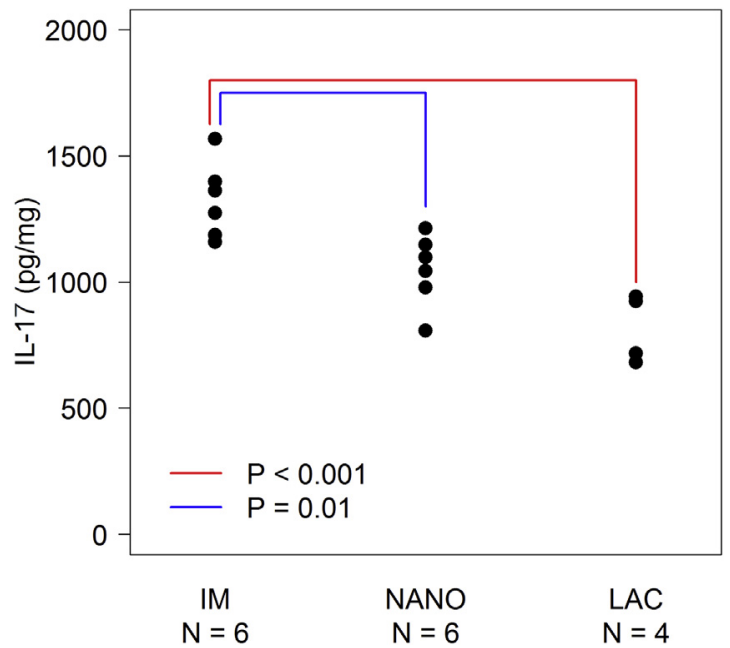

FIGURE 6. Scatter plot graph illustrating elevated IL-17 levels in the IM group compared with the NANO and LAC groups. $I L$, Interleukin; $I M$, intramuscular; $N A N O$, nanoparticle; $L A C$, lactose. 

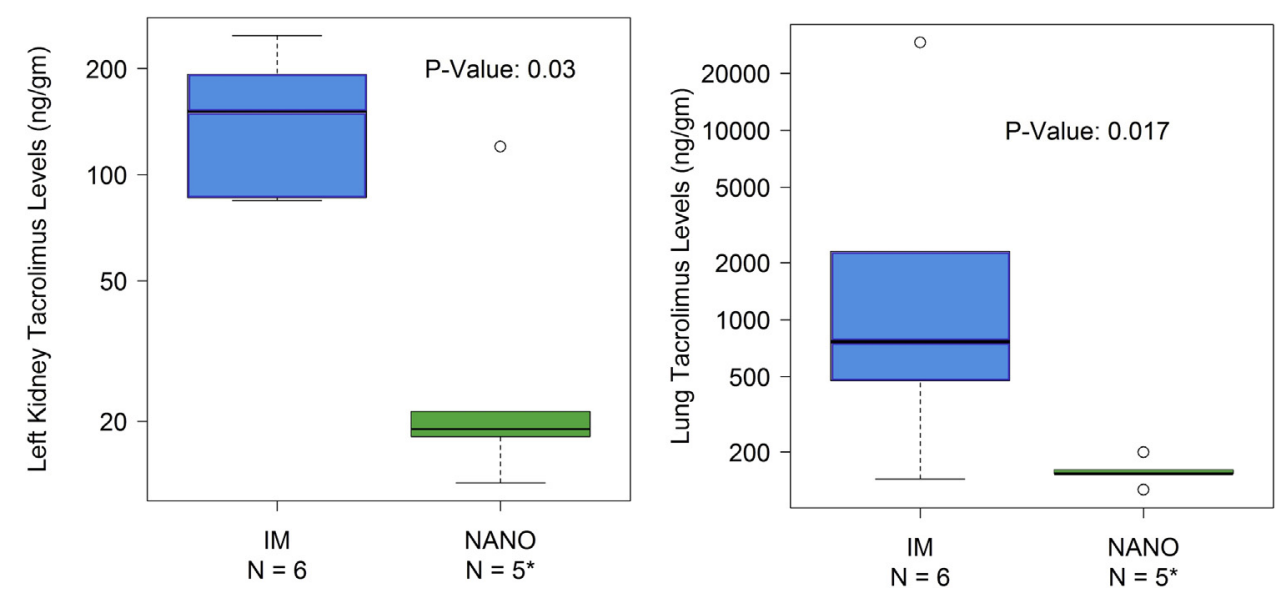

FIGURE 7. Box-whisker diagram of the tacrolimus drug concentrations in the tissue: kidney (left) and lung allograft (right). IM, Intramuscular; $N A N O$, nanoparticle.

for tacrolimus, which was shown to have maximum protection ${ }^{10}$ and chose the twice daily inhaled dosing of $6.4 \mathrm{mg}$ nTAC to maximize the therapeutic effect. The trough levels in the lung allograft were less than $200 \mathrm{ng} / \mathrm{g}$ (Figure 7), and conceivably our peak concentration may have been higher than $270 \mathrm{ng} / \mathrm{g}$ as intended. In addition, when tacrolimus levels in the lung allografts are compared, the inhaled group achieved similar antirejection at a lower level (Figure 7, right). This is an intriguing finding and merits further studies to evaluate the mechanism of action and how the 2 routes of administration may target different cell populations or alter unrelated pathways to achieve similar results. It could also be related to making the active drug available at the site of action when delivered by inhalation. Future studies could be designed to evaluate the response and safety of larger dose of inhaled nTAC to reach higher lung tissue concentrations and further reduce the rejection in the allograft. Ingu and colleagues ${ }^{14}$ have elucidated a possible dose-dependent response in their animal study, and it can be extrapolated that antirejection benefit can be enhanced by increasing the dose of nTAC. In vitro studies indicate that pulmonary epithelial cells are tolerant to nTAC even at several times the therapeutic concentrations. ${ }^{15}$ We have prior studies in healthy rats that inhaled high-dose tacrolimus for 28 days with no evidence of pulmonary, renal, or hepatic injury by both biochemical analysis and histopathology. ${ }^{16}$ This study focused on evaluating a single drug therapy (tacrolimus) in preventing acute rejection after lung transplant rather than standard triple immunosuppression to allow for direct comparison with prior studies. ${ }^{10}$

Another novelty of our study is the use of the Aperio Digital Pathology Solutions to evaluate the rejection in the lung allografts and allows for objective digital examination of the slides using proprietary software for cellular infiltrates. A pulmonary pathologist assessed all components of the lung parenchyma (bronchial, bronchiolar, vascular, and alveolar space) for cellular infiltrates to come up with a more composite evaluation of rejection: the modified pathology score. The modified pathology score has been internally validated by a panel of pathologists who demonstrated agreement with scoring pathologic lesions in a blinded fashion.

In the cytokine assays, we did see a statistically significant attenuation of proinflammatory cytokines in both our treatment groups (IM and NANO). However, IL-12 was surprisingly elevated in the NANO group (Figure 5), whereas IL-17 showed an increase in the IM group (Figure 6). The importance of these findings is unclear, but higher levels of both IL-12 and IL-17 appear to be associated with less acute rejection and may point toward an unknown feedback mechanism meriting further evaluation.

\section{CONCLUSIONS}

Inhaled nTAC given as twice-daily treatment reduced rejection to the same extent as systemic tacrolimus (IM), with lower systemic levels of the drug and at lower allograft concentrations of the treatment drug.

\section{Conflict of Interest Statement}

Authors have nothing to disclose with regard to commercial support.

The authors thank the Core for Advanced Translational Technologies at the University of Texas Health Science Center at San Antonio and Dr Carolina Livi for Luminex service to perform cytokine measurements. The authors also thank Dr Joel E. Michalek, Benjamin Ehler, and Christopher Louden, of the Department of Epidemiology and Biostatistics at the University of Texas Health Science Center at San Antonio, for help with statistical analysis and representation of our data.

\section{References}

1. Yusen RD, Edwards LB, Dipchand AI, Goldfarb SB, Kucheryavaya AY, Levvey BJ, et al. The Registry of the International Society for Heart and Lung Transplantation: Thirty-third Adult Lung and Heart-Lung Transplant 
Report-2016; Focus Theme: Primary Diagnostic Indications for Transplant. $J$ Heart Lung Transplant. 2016;35:1170-84.

2. Burton CM, Iversen M, Carlsen J, Mortensen J, Andersen CB, Steinbrüchel D, et al. Acute cellular rejection is a risk factor for bronchiolitis obliterans syndrome independent of post-transplant baseline FEV1. J Heart Lung Transplant. 2009; 28:888-93.

3. Keller CA, Cagle PT, Brown RW, Noon G, Frost AE. Bronchiolitis obliterans in recipients of single, double, and heart-lung transplantation. Chest. 1995;107:973-80.

4. Bhorade SM, Stern E. Immunosuppression for lung transplantation. Proc Am Thorac Soc. 2009;6:47-53.

5. Iacono AT, Johnson BA, Grgurich WF, Youssef JG, Corcoran TE, Seiler DA, et al. A randomized trial of inhaled cyclosporine in lung-transplant recipients, N Engl J Med. 2006;354:141-50.

6. Keenan RJ, Iacono A, Dauber JH, Zeevi A, Yousem SA, Ohori NP, et al. Treatment of refractory acute allograft rejection with aerosolized cyclosporine in lung transplant recipients. J Thorac Cardiovasc Surg. 1997;113:335-41.

7. Sinswat P, Overhoff KA, McConville JT, Johnston KP, Williams RO III. Nebulization of nanoparticulate amorphous or crystalline tacrolimus-single-dose pharmacokinetics study in mice. Eur J Pharm Biopharm. 2008;69:1057-66.

8. Watts AB, Cline AM, Saad AR, Johnson SB, Peters JI, Williams RO III. Characterization and pharmacokinetic analysis of tacrolimus dispersion for nebulization in a lung transplanted rodent model. Int J Pharm. 2010;384:46-52.

9. Mizuta T, Kawaguchi A, Nakahara K, Kawashima Y. Simplified rat lung transplantation using a cuff technique. J Thorac Cardiovasc Surg. 1989;97:578-81.

10. Ide N, Nagayasu T, Matsumoto K, Tagawa T, Tanaka K, Taguchi T, et al. Efficacy and safety of inhaled tacrolimus in rat lung transplantation. J Thorac Cardiovasc Surg. 2007; 133:548-53.
11. Stewart S, Fishbein MC, Snell GI, Berry GJ, Boehler A, Burke MM, et al Revision of the 1996 working formulation for the standardization of nomenclature in the diagnosis of lung rejection. J Heart Lung Transplant. 2007;26: $1229-42$.

12. Levine S. Safety and tolerability of single dose inhaled tacrolimus in healthy subjects. Chest. 2011;140(4_MeetingAbstracts):1020A.

13. Bayer J, Das NA, Baisden CE, Rani M, DeArmond DT, Peters JI, et al. Effect of inhaled tacrolimus on ischemia reperfusion injury in rat lung transplant model. $J$ Thorac Cardiovasc Surg. 2013;146:1213-9.

14. Ingu A, Komatsu K, Ichimiya S, Sato N, Hirayama Y, Morikawa M, et al. Effects of inhaled FK 506 on the suppression of acute rejection after lung transplantation: use of a rat orthotopic lung transplantation model. J Heart Lung Transplant. 2005;24:538-43.

15. Deuse T, Blankenberg F, Haddad M, Reichenspurner H, Phillips N, Robbins RC, et al. Mechanisms behind local immunosuppression using inhaled tacrolimus in preclinical models of lung transplantation. Am J Respir Cell Mol Biol. 2010;43: 403-12.

16. Watts AB, Peters JI, Talbert RL, O’Donnell KP, Coalson JJ, Williams RO III. Preclinical evaluation of tacrolimus colloidal dispersion for inhalation. Eur J Pharm Biopharm. 2011;77:207-15.

Key Words: regional immunosuppression, tacrolimus, inhaled nanoparticle, orthotopic rat lung transplant, modified pathology score for rejection in rats, cytokine profile in acute rejection 
TABLE E1. Data

\begin{tabular}{|c|c|c|c|c|c|c|c|c|c|c|}
\hline \multirow[b]{2}{*}{ Name } & \multirow[b]{2}{*}{ Group } & & \multicolumn{3}{|c|}{ Tacrolimus levels } & \multicolumn{5}{|c|}{ Pathology } \\
\hline & & & Blood TAC & $\begin{array}{c}\text { Allograft } \\
\text { TAC (ng/g) }\end{array}$ & $\begin{array}{c}\text { Right kidney } \\
\text { TAC (ng/g) }\end{array}$ & Bronchus & Bronchiole & Vessels & $\begin{array}{c}\text { Alveolar } \\
\text { space cellularity }\end{array}$ & Sum \\
\hline 93 & LAC & & - & - & - & 3 & 3 & 3 & 2 & 11.00 \\
\hline 94 & LAC & & - & - & - & 3 & 3 & 3 & 2 & 11.00 \\
\hline 95 & LAC & & - & - & - & 3 & 3 & 3 & 2.5 & 11.50 \\
\hline 96 & LAC & & - & - & - & 3 & 3 & 3 & 3 & 12.00 \\
\hline Mean & & Mean & - & - & - & 3.00 & 3.00 & 3.00 & 2.38 & 11.38 \\
\hline Median & & Median & - & - & - & 3.00 & 3.00 & 3.00 & 2.25 & 11.25 \\
\hline $\mathrm{SD}$ & & SD & - & - & - & 0.00 & 0.00 & 0.00 & 0.48 & 0.48 \\
\hline 115 & IM & & 1.191720783 & 143.98 & 45.33 & 2 & 2 & 2 & 0 & 6.00 \\
\hline 117 & $\mathrm{IM}$ & & 2.729957202 & 755.93 & 82.61 & 2 & 0.75 & 1 & 0 & 3.75 \\
\hline 121 & IM & & 5.186019902 & 773.87 & 152.62 & 0.75 & 0.5 & 0.5 & 0 & 1.75 \\
\hline 123 & $\mathrm{IM}$ & & 10.17160738 & 2297.42 & 119.86 & 1 & 0.5 & 1 & 0 & 2.50 \\
\hline 130 & IM & & 8.651374722 & 29086.51 & 212.71 & 0.75 & 1 & 1 & 0 & 2.75 \\
\hline 133 & IM & & 4.3647071 & 476.52 & 117.41 & 1.5 & 1 & 1 & 0 & 3.50 \\
\hline Mean & & Mean & 5.38 & 5589.04 & 121.76 & 1.33 & 0.96 & 1.08 & 0.00 & 3.38 \\
\hline Median & & Median & 4.78 & 764.90 & 118.64 & 1.25 & 0.88 & 1.00 & 0.00 & 3.13 \\
\hline SD & & SD & 3.44 & 11535.14 & 57.66 & 0.58 & 0.56 & 0.49 & 0.00 & 1.47 \\
\hline 120 & NANO & & 1.856434464 & 154.37 & 37.28 & 0.5 & 0.75 & 2 & 0 & 3.25 \\
\hline 122 & NANO & & 1.317330182 & 126.21 & 30.46 & 0.75 & 2 & 2 & 0 & 4.75 \\
\hline 125 & NANO & & 1.129413943 & 199.5 & 29.18 & 0.5 & 0.75 & 1 & 0 & 2.25 \\
\hline 126 & NANO & & 1.662534452 & 160.6 & 24.95 & 1.5 & 2 & 1.5 & 0 & 5.00 \\
\hline 132 & NANO & & 1.58332995 & 153.49 & 25.23 & 1.5 & 2 & 3 & 0 & 6.50 \\
\hline 134 & NANO & & 1.144793141 & 68.37 & 28.64 & 1.5 & 2 & 2.5 & 0 & 6.00 \\
\hline Mean & & Mean & 1.45 & 143.76 & 29.29 & 1.04 & 1.58 & 2.00 & 0.00 & 4.63 \\
\hline Median & & Median & 1.45 & 153.93 & 28.91 & 1.13 & 2.00 & 2.00 & 0.00 & 4.88 \\
\hline SD & & SD & 0.30 & 43.79 & 4.49 & 0.51 & 0.65 & 0.71 & 0.00 & 1.62 \\
\hline
\end{tabular}

TAC, Tacrolimus; $L A C$, lactose; $S D$, standard deviation; $I M$, intramuscular; $N A N O$, nanoparticle. 